\title{
A Retrospective Study on Clinical Significance of Imaging And Endobronchial Features In Pulmonary Lymphoma Diagnosed By Bronchoscopy: When Should We Perform Bronchoscopy?
}

Shao-Ting Wang ( $\sim$ bird0162@163.com )

Peking Union Medical College Hospital

Research Article

Keywords: pulmonary lymphoma, Chest CT, bronchoscopy

Posted Date: April 22nd, 2021

DOl: https://doi.org/10.21203/rs.3.rs-439281/v1

License: (9) This work is licensed under a Creative Commons Attribution 4.0 International License. Read Full License 


\section{Abstract}

Background: Pulmonary lymphoma is rare while the radiographic lesions are nonspecific. The use of bronchoscopy, is controversial, and may be of limited diagnostic value for pulmonary lymphoma diagnosis.

Methods: Cases of pulmonary lymphoma diagnosed by bronchoscopy were retrospectively reviewed from January 2010 till December 2020. Clinico-radiological records of these cases were retrieved and bronchoscopy was performed with histopathological evaluation of the biopsy specimens.

Results: Of the 41 cases included in our study, 20 were primary pulmonary lymphoma. The majority of subtypes were diffuse large B-cell lymphoma(43.9\%) and extranodal marginal zone lymphoma of mucosa-associated lymphoid tissue lymphoma(29.3\%), with high percentage of underlying chronic disease. The common Computed Tomography(CT) abnormalities were mass(43.9\%), consolidation(24.4\%) or ground-glass opacities(24.4\%). To evaluate the pulmonary lesions distribution, $41.5 \%$ patients had segmental lesions, $29.3 \%$ presences of diffuse lesions, $17.1 \%$ of local lesions and $12.2 \%$ of hilar lesions, with the maximal value of standard uptake value $\left(S U V_{\max }\right)$ median value of 9.6. At bronchoscopy, $41.5 \%$ lesions were identified as focal solitary mass, $22 \%$ were normal, $17.1 \%$ were mucosae asperity or edema, $9.8 \%$ were diffuse airway submucosal nodules or thickening and $9.8 \%$ were bronchiole stenosis, while certain endobronchial patterns may correspond to specific imaging features.

Conclusion: The imaging features and endobronchial patterns of pulmonary lymphoma are characteristic. Bronchoscopy is still a useful tool, that is minimally invasive, to diagnose pulmonary lymphoma, while accurate analysis of the clinico-radiological records is essential before such procedure.

\section{Background}

Pulmonary lymphoma is involved in $<10 \%$ of Hodgkin lymphomas and $<5 \%$ of non-Hodgkin lymphomas. The radiographic patterns in the lung include nodular, patchy pneumonic infiltrates, mass, lymphomatous lesions, or military hematogenous forms[1]. Lymphoma manifesting such as lung opacities is sometimes difficult to diagnose bronchoscopically, that Computed Tomography(CT) guided transthoracic needle biopsy or even thoracoscopy has been described as a useful modality for obtaining larger biopsy samples in diffuse parenchymal lung lesions. However, pulmonary physicians still tend to perform bronchoscopy as the first step on patients of suspected pulmonary lymphoma with certain CT imaging features. Are there any specific imaging features in patients diagnosed under bronchoscopy? What are their corresponding endobronchial lesions under bronchoscopy? In this study, we retrospectively analyze the clinical manifestation and features of patients bronchoscopically diagnosed as pulmonary lymphoma.

\section{Methods}




\section{Patients}

A total of 41 patients with pulmonary lymphoma diagnosed by bronchoscopy were retrospectively identified through key words search (bronchoscopy, lymphoma) of pathological databases at Peking Union Medical College Hospital for clinical assessment (PUMCH, Beijing, China), who were diagnosed between January 1, 2010, and December 31,2020. The study protocol was reviewed and approved by the ethical committee of Peking Union Medical College Hospital, and written consent was obtained from all subjects.

\section{Imaging Protocol}

All patients were scanned using axial CT was performed (140 kV, $35 \mathrm{~mA}$, pitch of 1:1, 5-mm thickness with 3-mm gap, $512 \times 512$ matrix, $70-\mathrm{cm}$ field of view,6msv) from the neck to the mid-thigh performed in inspiratory apnoea before the bronchoscopy. Precontrast and enhanced images were obtained in 16 patients. As previous study mentioned[2], features of the pulmonary lesions were described as localization (bilateral or unilateral), distribution (local, segmental, diffuse or hilar) and morphological pattern (consolidation, mass, nodule, or ground-glass opacities(GGO), et al). Area of consolidation was defined as homogeneous increase in pulmonary parenchymal attenuation obscuring the vessels margins and airways walls; mass was defined as rounded well-defined or moderately well-defined opacity greater than $3 \mathrm{~cm}$ in maximum diameter; nodule was defined as rounded well-defined or moderately well-defined opacity equal to or less than $3 \mathrm{~cm}$ in maximum diameter; GGO was defined as a hazy increased attenuation of lung with preservation of bronchial and vascular margins, and mosaic pattern was a condition in which there was a patchwork of regions of different lung attenuation on thin-section CT. Other CT findings, including air bronchogram, bronchiectasis, cavitation, thoracic lymphadenopathy, pleural effusion, calcification, pulmonary cysts, were also recorded.

A total of 21 patients had undergone fluorine18 2deoxy2fluoroDglucose (18FFDG) Positron Emission Computed Tomography(PET) scanning. PET/CT scans were performed on the PET/CT system (Biograph 64 TruePoint; Siemens, Knoxville, Tennessee, USA). The maximal value of standard uptake value $\left(\mathrm{SUV}_{\max }\right)$ in pulmonary lesions was recorded.

\section{Bronchoscopy}

All patients underwent a diagnostic bronchoscope(Table 3). Among 41 patients, right lower lobe(24.4\%), left upper lobe(19.5\%) and left lower lobe(19.5\%) were the most frequently examined location, as physicians prefer to perform TBLB in the lower lobe, especially patients with normal endobronchial pattern. A total of 35 pulmonary lesions were identified: 17 areas of focal solitary mass, 7 mucosae asperity or edema, 4 diffuse airway submucosal nodules or thickening, 4 bronchiole stenosis and 3 only lymph node enlargement(Fig. 1), while stations $4 \mathrm{R}$ and 7 were the most frequently examined lymph node. 
One bleeding within 48 hours after neoplasm biopsy as procedure-related complication occurred in this study.

\section{Diagnosis}

Diagnosis of lymphoma was according the updated WHO classification of Lymphoid Malignancies by PUMCH pathology department. Some diagnosis were established by MDT(Multiple Disciplinary Team) with experienced hematopathologist with the help of immunohistochemistry or even flow cytometry[3]. While primary pulmonary lymphoma (PPL) is defined as a lymphoma confined to the lung with or without hilar lymph node involvement at the time of diagnosis or up to 3 months thereafter[1,4].

\section{Statistical analysis}

Statistical analyses were processed by statistical software program (PASW statistics 16; SPSS Inc., Chicago, IL., USA). Means and percentages were presented as appropriate.

\section{Results}

\section{Patients}

All 41 patients were histologically confirmed with pulmonary lymphoma. The female: male ratio is 1:1.1, while median age is 52 years(range 2277 years). All patients underwent a diagnostic bronchoscope biopsy procedure(including 7 patients underwent TBNA also). Among 41 patients, 3 underwent following cervical or axillary lymph node biopsy, 2 underwent parotid gland biopsy, 1 underwent gastroscopy; 3 underwent a CTguided transthoracic needle biopsy procedure to confirm the subtype classification or evaluate the neoplasm stage. A total of 20 patients were diagnosed with PPL. Among 41 patients, 18 cases were regarded as diffuse large B-cell lymphoma(DLBCL), 12 were extranodal marginal zone lymphoma of mucosa-associated lymphoid tissue lymphoma(MALT), 5 were Hodgkin lymphoma(HL) (Table 1). The predominant symptoms experienced by patients were coughing (36.6\%), fever (17.1\%), dyspnea (22\%) and, while $48.8 \%$ patients suffered with symptom B. An interesting finding is high percentage of underlying chronic disease (chronic hepatitis B $12.2 \%$, chronic hepatitis C $7.3 \%$ and Sjögren's syndrome 9.8\%), which are summarized in Table 1. 
Patient characteristics.

\section{Characteristics No(\%)}

No. of patients 41 (include PPL 20(48.8),SPL 21(51.2))

$\begin{array}{ll}\text { Male/female } & 24 / 17 \\ \begin{array}{l}\text { Age, median, } \\ \text { year[range] }\end{array} & 52[22-77]\end{array}$

$\begin{array}{lllll}\text { Underlying } & \text { Sjögren's } & \text { Chronic hepatitis } & \text { Chronic hepatitis C 3(7.3) } & \text { Other } \\ \text { chronic } & \text { syndrome } & \text { B 5(12.2) } & & \text { solid } \\ \text { disease } & 4(9.8) & & & \text { tumor } \\ & & & 3(7.3)\end{array}$

$\begin{array}{llll}\text { Diagnosis } & \text { DLBCL } & \text { HL 5(12.2) } & \text { MALT 12(29.3) } \\ & \text { 18(43.9) } & & \text { Other\& } \\ & & & \end{array}$

$\begin{array}{lll}\text { Stage }^{*} \quad \text { III 2(4.9) IVA 7(17.1) IVB 18(43.9) } & \text { II) }\end{array}$

\begin{tabular}{llllll}
$\begin{array}{l}\text { Chief } \\
\text { Complaint }\end{array}$ & $\begin{array}{l}\text { Cough } \\
15(36.6)\end{array}$ & Fever 7(17.1) & Dyspnea 9(22.0) & \multicolumn{1}{c}{$\begin{array}{l}\text { Other } \\
10(24.4)\end{array}$} \\
\hline Symptom B & $20(48.8)$ & & & \\
\hline Therapy & $\begin{array}{l}\text { Chemotherapy } \\
27(65.9)\end{array}$ & $\begin{array}{l}\text { Chemo-and } \\
\text { radiotherapy } \\
1(2.4)\end{array}$ & $\begin{array}{l}\text { Watch\&Wait } \\
2(4.9)\end{array}$ & $\begin{array}{l}\text { Chemotherapy } \\
\text { and ASCT }\end{array}$ & $\begin{array}{l}\text { Unknown } \\
8(19.5)\end{array}$ \\
\hline
\end{tabular}

PLL: primary pulmonary lymphoma; SPL: secondary pulmonary lymphomas; ASCT: autologous stem cell transplantation * 14 patients fell to evaluate the stage due to personal willingness or poor general condition; DLBCL: diffuse large B-cell lymphoma; HL:Hodgkin lymphoma; MALT: extranodal marginal zone lymphoma of mucosa-associated lymphoid tissue lymphoma; ${ }_{\text {including anaplastic large cell }}$ lymphoma, high-grade B-cell lymphoma, small lymphocytic non-Hodgkin's lymphoma or not specified subtype classification.

\section{Imaging features}

CT. Pulmonary lesions were identified in all patients: 26 (63.4\%) presenting as bilateral pulmonary lesions. The most common CT abnormality was a mass(43.9\%), consolidation(24.4\%) or GGO(24.4\%), while thoracic lymphadenopathy was observed in 14 of 41 patients (34.1\%), air bronchogram in the 6 (14.6\%) patients and pleural effusion in the $6(14.6 \%)$ patients. 4 patients with pulmonary cysts coexisted with Sjögren's syndrome or chronic hepatitis B. Evaluating pulmonary lesions distribution, $41.5 \%$ patients had segmental lesions, $29.3 \%$ presences of diffuse lesions, $17.1 \%$ of local lesions and $12.2 \%$ hilar lesions presentation.

PETCT. A total of 21 patients underwent PETCT scanning, including 6 in the PPL group and 15 in the SPL group. Only one patient, presenting with diffuse pulmonary GGO and another patient, presenting with local lesion in the ostium of left upper lobe, were PET-CT negative. The extrapulmonary lymphadenopathy 
of SPL involved neck, stomach, liver, spleen, intestine and pelvic regions, et al. The SUV $\mathrm{max}_{\text {of }}$ the pulmonary lesions ranged between 0 and 27.9 , with 9.6 as the median value(Table 2).

Table 2

Imaging features.

\begin{tabular}{|c|c|}
\hline Imaging features & No(\%) \\
\hline \multicolumn{2}{|l|}{ Location } \\
\hline Unilateral & $15(36.6)$ \\
\hline Bilateral & $26(63.4)$ \\
\hline \multicolumn{2}{|l|}{ Distribution } \\
\hline Local & $7(17.1)$ \\
\hline Segmental & $17(41.5)$ \\
\hline Diffuse & $12(29.3)$ \\
\hline Hilar & $5(12.2)$ \\
\hline \multicolumn{2}{|l|}{ Morphological pattern } \\
\hline Consolidation & $10(24.4)$ \\
\hline Mass & 18(43.9) \\
\hline Nodule & $3(7.3)$ \\
\hline GGO & $10(24.4)$ \\
\hline \multicolumn{2}{|l|}{ Secondary associated features } \\
\hline Air bronchogram & $6(14.6)$ \\
\hline Bronchiectasis & $2(4.9)$ \\
\hline Cavitation & $3(7.3)$ \\
\hline Pleural effusion & $6(14.6)$ \\
\hline Pulmonary cysts & $4(9.8)$ \\
\hline Thoracic lymphadenopathy & 14(34.1) \\
\hline Interlobular septa thicken & $2(4.9)$ \\
\hline $\mathrm{SUV}_{\text {max }}$, median[range] & $9.6[0-27.9]$ \\
\hline
\end{tabular}




\section{Bronchoscopy features}

Location of lesion examined

Right lung

RUL:RML:RLL: Right main bronchus: Right intermediate bronchus

Left lung

LUL:LLL: Left main bronchus

$4 R: 5: 7$

No. samples per patient(median,[range])

Endobronchial pattern

Diffuse airway submucosal nodules or thickening

Focal solitary mass

Mucosae asperity or edema

Bronchiole stenosis

Only lymph node enlargement

Normal

RUL: right upper lobe; RML: right medium lobe; RLL: right lower lobe; LUL: left upper lobe; LLL: left lower lobe

\section{Discussion}

Pulmonary lymphoma is rare, while the most common pathological type of PLL is MALT lymphoma or low-grade marginal zone B-cell lymphoma, and other types include DLBCL, follicular lymphoma, Burkitt lymphoma, T-cell lymphoma, and et al. For SPL, DLBCL accounts for around $36 \%$, MALT for $23 \%$, while HL for $15 \%[5]$. MALT lymphoma is believed to arise as a result of chronic inflammation of the marginal zone B cells of the "bronchial-associated lymphoid tissue". It may associate with autoimmune disorders including Sjögren's syndrome, rheumatoid arthritis, systemic lupus erythematosus, Hashimoto thyroiditis, chronic hepatitis, Helicobacter pylori infection or even smoke. Pulmonary DLBCL can be transformed from MALT, which is much more aggressive and tends to manifest with systemic symptoms $B[6,7]$.

The most common pathological diagnosis in our study is DLBCL, MALT and HL. Our study also showed high morbidity of chronic hepatitis $B$, chronic hepatitis $C$ and Sjögren's syndrome in patients with pulmonary lymphoma, as 3 out of 4 Sjögren's syndrome patients were pathologically diagnosed as MALT. 
Pulmonary lymphoma has specific radiographic patterns and endobronchial changes. Rose described two distinct patterns of lymphomatous involvement: Type 1 occurs in patients with clinically apparent systemic lymphoma, whose common symptom is pneumonitis. The airway involvement is diffuse submucosal nodules in airway, while other radiographic findings include parenchymal infiltrates, mediastinal lymphadenopathy. Some authors have suggested a preferential distribution at airway bifurcations[8]. Type 2 consists of a solitary mass involving the central airways without evidence of systemic lymphoma, while the symptoms are airway obstruction, wheezing or cough. Other radiographic findings include atelectasis, lymph node enlargement in proximity[9].

Previous studies showed the most frequent patterns are consolidations $(\sim 55 \%)$, nodules $(\sim 55 \%)$ and masses ( $\sim 50 \%)$, and $\sim 85 \%$ of the patients have airways within the lesions. Micronodules ( $20 \%)$, ground-glass opacities ( $25 \%)$ and septal lines $(\sim 10 \%)$ are less frequent. Hilar or mediastinal lymphadenopathy may be found in $\sim 15 \%$ of cases, which are usually $<1.5 \mathrm{~cm}$ and a mild pleural effusion in $\sim 10 \%$ of cases $[1,10,11]$.

In our study, nearly half patients are type 1 , that the major CT abnormality was consolidation accounts for $24.4 \%$, and GGO accounts for $24.4 \%$, may involve parenchymal and lymphomatous infiltrates. (1)As Fig. 1a,b showed, for consolidation lesions, endobronchial patterns under bronchoscopy are usually mucosae asperity or edema, occasionally focal solitary mass. The air bronchogram sign and angiogram sign are more characteristic manifestations of this disease. Its pathological basis is that the neoplasm originated from pulmonary interstitium, while the pulmonary blood vessels still remain. The neoplasm grows along the bronchial wall, so the original bronchial remains within the lymphoma. It was previously noted that MALT are associated with air bronchogram in nearly $50 \%$ of cases[1]. While the hyperplasia of fibrous tissue can lead to traction bronchiectasis, that in consolidation lesions, bronchiectasis is another feature in pervious study[12]. (2)For GGO lesions, endobronchial patterns under bronchoscopy are always normal(except only one case found focal solitary mass under bronchoscopy), as Fig. 1c,d showed, while TBLB can usually make the histological diagnosis. Another half patients are type 2 , that the major CT abnormality was mass accounts for $43.9 \%$, and nodule accounts for $7.3 \%$. (3)For mass lesions, with or without cavitation, endobronchial patterns under bronchoscopy are diffuse airway submucosal nodules or thickening, frequently focal solitary mass, as Fig. 1e,f showed. It is considered that cavitary lesions suggest a higher grade lymphoma, while consolidation suggests a lower grade lymphoma with reserved bronchial tree. (4)For nodule lesions, endobronchial patterns under bronchoscopy are bronchiole stenosis, as Fig. 1g,h,i showed, frequently mucosae asperity or edema. We found bronchial obstruction and associated atelectasis, ultimately atelectasis is usually suggestive of concomitant endobronchial disease. It seems the frequency of nodule lesions is much lower than previous studies mentioned above, because nodules are always not accessible by bronchoscopy. However, there is tendency for these CT or endobronchial patterns to overlap, and patients may present with several simultaneously.

Since 2008 World Health Organization classification, subtype classification of lymphoma is necessary for an accurate diagnosis, to predict disease prognosis, and to guide the treatment[13]. Our study excluded cases of suspected pathological diagnosis of lymphoma, but included 7 cases without subtype 
classification, that they needed following extrapulmonary biopsy. Previous studies suggested bronchoscopy may be of limited diagnostic value for pulmonary lymphoma diagnosis[14]. For example, Ferraro et al. reported that bronchoscopy was performed in 39 patients with PPL but confirmed the diagnosis in only seven patients (18\%)[15]. The sensitivity of bronchial and transbronchial biopsies in detecting MALT lymphoma have been reported to be 30 and $88 \%$, respectively in different researches $[1$, 16].

However, for EBUS-TBNA, the diagnostic sensitivity seems better: the diagnostic sensitivity in lymphoma varied between $38 \%$ and $91 \%$ in the previous studies, according to diverse diagnostic criteria of the individual studies, proportion of new or prior lymphoma, prevalence of subtypes and implementing ancillary methods $[17,18]$. For example, involved 65 cases with final diagnosis of lymphoma, lqbal et al. indicated that the diagnostic sensitivity of EBUS-TBNA for patients with and without previously diagnosed lymphoma was $55 \%$ and $22 \%$, respectively[18]. Other researches studies the use of new techniques as transbronchial needle forceps or ROSE, indicating the benefit that the lymphoma diagnosis undertaken by an expert group working closely with lymphoma pathologists and a lymphoma MDT[19, 20]. Gonzalo et al. revealed the result of a meta-analysis, which involved fourteen studies with 425 patients. EBUS-TBNA reported an overall sensitivity of $66.2 \%$ and specificity of $99.3 \%$. For a new diagnosis of lymphoma, thirteen studies including 243 participants reported sensitivity of $67.1 \%$ and specificity of $99.6 \%$; for recurrence of lymphoma, eleven studies included 166 participants reported sensitivity of $77.8 \%$ and specificity of $99.5 \%$. In the recurrence group, the use of ROSE, sample size and flow cytometry was found to increase the sensitivity of EBUS-TBNA, albeit a potential sources of heterogeneity[21].

Our study included definitive diagnosis of lymphoma under bronchoscopy, that only 7 patients underwent TBNA meanwhile, probably because of the low sensitivity of EBUS-TBNA without ROSE. From my point of view, the use of bronchoscopy to diagnose lymphoma is easy to perform and minimally invasive with low frequency of procedure-related complication. Approximately one-third of patients can be given a definitive diagnosis of lymphoma, with carefully patients selected according to CT features mentioned above(mass, GGO or consolidation), avoiding the need for further invasive procedures, such as CTguided transthoracic needle biopsy or thoracoscopy. Second, with high diagnostic accuracy in both benign and malignant diseases, bronchoscopy with or without EBUS-TBNA provides high differential diagnostic yield for lesions mimicking lymphoma such as lung cancer, infection and sarcoidosis[22]. Third, either a definitive diagnosis or suspected lymphoma was provided currently for $85 \%$ of patients, which may be helpful for decisions with the management or further diagnostic procedures.

\section{Conclusions}

Pulmonary lymphoma presents distinct features in CT scan, which may indicate certain endobronchial patterns under bronchoscopy. The closer observation of CT images may facilitate the differential diagnosis and help in carefully patients selecting for bronchoscopy. This first-line procedure may improve 
the early diagnosis and guide further individual clinical evaluation, aiding the early initiation of therapy strategies.

\section{Abbreviations}

CT: Computed Tomography; SUVmax: maximal value of standard uptake value; GGO: ground-glass opacities; 18F FDG: fluorine 182 deoxy 2 fluoro D glucose; PET: Positron Emission Computed Tomography; ROSE: rapid on-site cytological examination; TBLB: transbronchial lung biopsy; EBUS: endobroncheal ultrasonography; TBNA: transbronchial needle aspiration; MDT: Multiple Disciplinary Team; PPL: primary pulmonary lymphoma; DLBCL: diffuse large B-cell lymphoma; MALT: extranodal marginal zone lymphoma of mucosa-associated lymphoid tissue lymphoma; HL: Hodgkin lymphoma.

\section{Declarations}

\section{Acknowledgements}

The author would like to thank Jing Wang for her assistance in conducting the searching work in pathological databases at Peking Union Medical College Hospital.

\section{Authors' contributions}

S-T Wang designed the study, had full access to all the data in this study, performed statistical analysis and wrote the draft. All authors read and approved the final manuscript.

\section{Funding}

This research did not receive any specific grant from funding agencies in the public, commercial, or notfor-profit sectors.

\section{Availability of data and materials}

The datasets used for the current study are available from the corresponding author on reasonable request.

\section{Ethics approval and consent to participate}

This study was conducted in accordance with the amended Declaration of Helsinki. The ethical committee of Peking Union Medical College Hospital approved the protocol. Written informed consent was obtained from all subjects.

\section{Consent for publication}

Not applicable. 


\section{Competing interests}

The authors declare that they have no competing interests.

\section{References}

1. Cordier JF, Chailleux E, Lauque D, Reynaud-Gaubert M, Dietemann-Molard A, Dalphin JC, et al. Primary pulmonary lymphomas. A clinical study of 70 cases in nonimmunocompromised patients. Chest. 1993;103:201-8. doi: 10.1378/chest.103.1.201.

2. Austin JH, Muller NL, Friedman PJ, Hansell DM, Naidich DP, Remy-Jardin M, et al. Glossary of terms for CT of the lungs: recommendations of the Nomenclature Committee of the Fleischner Society. Radiology. 1996;200:327-31. doi: 10.1148/radiology.200.2.8685321.

3. Swerdlow SH, Campo E, Pileri SA, Harris NL, Stein H, Siebert R, et al. The 2016 revision of the World Health Organization classification of lymphoid neoplasms. Blood. 2016;127:2375-90. doi: 10.1182/blood-2016-01-643569.

4. Koss MN. Malignant and benign lymphoid lesions of the lung. Ann Diagn Pathol. 2004;8:167-87. doi: 10.1016/j.anndiagpath.2004.03.010.

5. William J, Variakojis D, Yeldandi A, Raparia K. Lymphoproliferative neoplasms of the lung: a review. Arch Pathol Lab Med. 2013;137:382-91. doi: 10.5858/arpa.2012-0202-RA.

6. Cadranel J, Wislez M, Antoine M. Primary pulmonary lymphoma. Eur Respir J. 2002;20:750-62. doi: 10.1183/09031936.02.00404102.

7. Pina-Oviedo S, Weissferdt A, Kalhor N, Moran CA. Primary Pulmonary Lymphomas. Adv Anat Pathol. 2015;22:355-75. doi: 10.1097/PAP.0000000000000090.

8. Gallagher CJ, Knowles GK, Habeshaw JA, Green M, Malpas JS, Lister TA. Early involvement of the bronchi in patients with malignant lymphoma. Br J Cancer. 1983;48:777-81. doi: 10.1038/bjc.1983.266.

9. Rose RM, Grigas D, Strattemeir E, Harris NL, Linggood RM. Endobronchial involvement with nonHodgkin's lymphoma. A clinical-radiologic analysis. Cancer. 1986;57:1750-5. doi: 10.1002/10970142(19860501)57:9<1750::aid-cncr2820570907>3.0.co;2-j.

10. Herbert A, Wright $D H$, Isaacson $P G$, Smith JL. Primary malignant lymphoma of the lung: histopathologic and immunologic evaluation of nine cases. Hum Pathol. 1984;15:415-22. doi: 10.1016/s0046-8177(84)80074-x.

11. Kennedy JL, Nathwani BN, Burke JS, Hill LR, Rappaport H. Pulmonary lymphomas and other pulmonary lymphoid lesions. A clinicopathologic and immunologic study of 64 patients. Cancer. 1985;56:539-52. doi: 10.1002/1097-0142(19850801)56:3<539::aid-cncr2820560322>3.0.co;2-d.

12. Hare SS, Souza CA, Bain G, Seely JM, Frcpc, Gomes MM, et al. The radiological spectrum of pulmonary lymphoproliferative disease. Br J Radiol. 2012;85:848-64. doi: 10.1259/bjr/16420165. 
13. Sverdlow SH CE, Harris NL. WHO classification of tumours of haemopoetic and lymphoid tissue. Lyon,France: IARC Press; 2008.

14. Xu HY, Jin T, Li RY, Ni YM, Zhou JY, Wen XH. Diagnosis and treatment of pulmonary mucosaassociated lymphoid tissue lymphoma. Chin Med J (Engl). 2007;120:648-51.

15. Ferraro P, Trastek VF, Adlakha H, Deschamps C, Allen MS, Pairolero PC. Primary non-Hodgkin's lymphoma of the lung. Ann Thorac Surg. 2000;69:993-7. doi: 10.1016/s0003-4975(99)01535-0.

16. Borie R, Wislez M, Thabut G, Antoine M, Rabbat A, Couderc LJ, et al. Clinical characteristics and prognostic factors of pulmonary MALT lymphoma. Eur Respir J. 2009;34:1408-16. doi: 10.1183/09031936.00039309.

17. Kennedy MP, Jimenez CA, Bruzzi JF, Mhatre AD, Lei X, Giles FJ, et al. Endobronchial ultrasoundguided transbronchial needle aspiration in the diagnosis of lymphoma. Thorax. 2008;63:360-5. doi: 10.1136/thx.2007.084079.

18. Iqbal S, DePew ZS, Kurtin PJ, Sykes AM, Johnson GB, Edell ES, et al. Endobronchial ultrasound and lymphoproliferative disorders: a retrospective study. Ann Thorac Surg. 2012;94:1830-4. doi: 10.1016/j.athoracsur.2012.08.051.

19. Herth FJ, Schuler H, Gompelmann D, Kahn N, Gasparini S, Ernst A, et al. Endobronchial ultrasoundguided lymph node biopsy with transbronchial needle forceps: a pilot study. Eur Respir J. 2012;39:373-7. doi: 10.1183/09031936.00033311.

20. Moonim MT, Breen R, Fields PA, Santis G. Diagnosis and subtyping of de novo and relapsed mediastinal lymphomas by endobronchial ultrasound needle aspiration. Am J Respir Crit Care Med. 2013;188:1216-23. doi: 10.1164/rccm.201303-04620C.

21. Labarca G, Sierra-Ruiz M, Kheir F, Folch E, Majid A, Mehta HJ, et al. Diagnostic Accuracy of Endobronchial Ultrasound Transbronchial Needle Aspiration in Lymphoma. A Systematic Review and Meta-Analysis. Ann Am Thorac Soc. 2019;16:1432-9. doi: 10.1513/AnnalsATS.201902-1750C.

22. Varela-Lema L, Fernandez-Villar A, Ruano-Ravina A. Effectiveness and safety of endobronchial ultrasound-transbronchial needle aspiration: a systematic review. Eur Respir J. 2009;33:1156-64. doi: 10.1183/09031936.00097908.

\section{Figures}




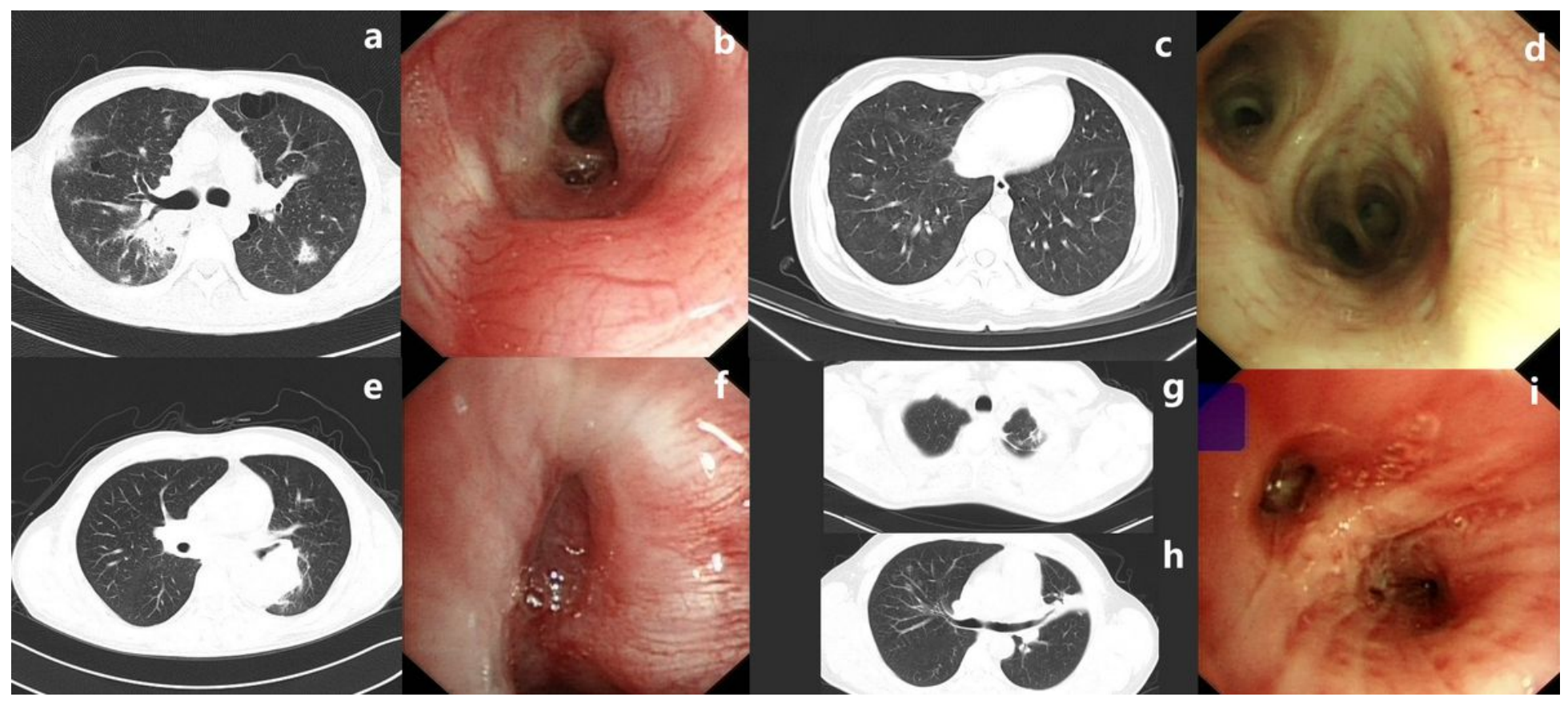

Figure 1

Chest Computed Tomography(CT) of patient No 1. shows consolidation of the right upper lobe of the lung with air bronchogram, with associated subpleural nodule and multiple pulmonary cysts. b.

Bronchoscopic findings of patient No 1. reveal mucosae hyperemia and edema in segment 2 of the right upper lobe. c. Chest CT of patient No 2. demonstrates bilateral multiple ground-glass opacities(GGO) lesions subpleural sparing. d. Bronchoscopic findings of patient No 2. are normal. e. Chest CT of patient No 3. reveals mass in segment 6 of the left lower lobe with bronchial obstruction. f. At bronchoscopy of patient No 3., a neoplasm is seen in segment 6 of the left lower lobe. g. Chest CT of patient No 4. shows multiple nodule of the left upper lobe. h. Chest CT of patient No 4. revealing atelectasis of the left upper lobe and multiple nodule of the right upper lobe. i. Bronchoscopic findings of patient No 4. demonstrate bronchial stenosis with mucosae edema of the right upper lobe. 